CONFORMAL GEOMETRY AND DYNAMICS

An Electronic Journal of the American Mathematical Society

Volume 3, Pages 79-101 (August 12, 1999)

S $1088-4173(99) 00031-4$

\title{
GEOMETRY OF THE FEIGENBAUM MAP
}

\author{
XAVIER BUFF
}

\begin{abstract}
We show that the Cvitanović-Feigenbaum equation can be interpreted as a linearizing equation, and the domain of analyticity of the Feigenbaum fixed point of renormalization as a basin of attraction. As a consequence, we give a combinatorial description of this ramified covering, and we show the surprising result that there exist points in the boundary of this domain with three accesses from inside the domain. Besides, there is a natural decomposition of this basin which makes it possible to recover a result of local connectivity by $\mathrm{Hu}$ and Jiang (The Julia set of the Feigenbaum quadratic polynomial is locally connected, Preprint, 1993) for the Feigenbaum Julia set.
\end{abstract}

We have included in this article several pictures. We would like to thank especially Louis Granboulan who helped us to draw some of the pictures using the program Pari, and Dan Sørensen who designed a wonderful program to draw Mandelbrot and Julia sets.

I am grateful to Adrien Douady for having taught me so much about holomorphic dynamics. Most of the tools to deal with the Feigenbaum equation were explained to me by Henri Epstein, and most of my ideas came during discussions with him in IHES, with Marguerite Flexor in Orsay and with John H. Hubbard in Cornell. I would also like to thank Adam Epstein, Christian Henriksen, Mikhail Lyubich and Carsten Petersen for several comments and suggestions. I am greatly indebted to these people.

\section{INTRODUCTION}

In 1976, Feigenbaum [F1] [F2], and independently Coullet and Tresser [CT] introduced the notion of renormalization for real dynamical systems. In 1985, the generalization to holomorphic dynamical systems via polynomial-like mappings, was introduced by Douady and Hubbard [DH].

In 1987, Lanford [L] gave a computer assisted proof of the existence of a fixed point for the period-doubling renormalization operator. His result was completed by Eckmann and Wittwer [EW]. Later, Epstein [E1] [E2] gave a proof of the existence which does not require the help of computers. Finally, in the 90's, Sullivan [S] proved the uniqueness of the fixed point for the period-doubling renormalization operator.

Received by the editors January 27, 1998 and, in revised form, May 19, 1999.

1991 Mathematics Subject Classification. Primary 58F; Secondary 30D05.

Key words and phrases. Dynamics, puzzle, renormalization, Feigenbaum, local connectivity.

(C)1999 American Mathematical Society 
This fixed point of renormalization satisfies a functional equation known as the Cvitanović-Feigenbaum equation:

$$
f(z)=-\frac{1}{\lambda} f \circ f(\lambda z), \quad 0<\lambda<1 .
$$

We will use this functional equation to study the domain of analyticity of the solution $f$. Epstein [E2] and McMullen [McM] (7.3) proved that it has a maximal analytic extension - still denoted by $f$ - to a simply connected, open set $\widehat{W}$, dense in $\mathbb{C}$. We will prove the following results.

Theorem A. The map $f_{\lambda}(z)=f(\lambda z)$ defined on $\widehat{W} / \lambda$ has a fixed point $x_{0} \in \widehat{W}$ with multiplier $-\lambda$. Besides, $f_{\lambda}$ is analytic on $\widehat{W}, f_{\lambda}(\widehat{W})=\widehat{W}$, and $\widehat{W}$ is the basin of attraction of the map $f_{\lambda}$. Finally, $f$ is a linearizer of the map $f_{\lambda}$.

Theorem B. All the critical points of $f$ are simple. The critical values of $f$ are all contained in the real axis. Besides, the analytic extension $f: \widehat{W} \rightarrow \mathbb{C}$ is a ramified covering, in the sense that for any bounded disk $\mathbb{D}_{R} \subset \mathbb{C}$, each component of $f^{-1}\left(\mathbb{D}_{R}\right)$ is proper over $\mathbb{D}_{R}$.

Since the critical values are real, we will then study the combinatorial structure of $f^{-1}(\mathbb{R})$. We will show that $f: \widehat{W} \rightarrow \mathbb{C}$ has the same combinatorial structure as the linearizer of a Blaschke product.

There was a belief that $\partial \widehat{W}$ was a Cantor bouquet. However, the following result proves that such is not the case.

Theorem C. The boundary of $\widehat{W}$ contains points with three accesses from inside $\widehat{W}$. In particular, this boundary does not have the structure of a Cantor Bouquet.

We will finally use a natural tiling of $\widehat{W}$ to study the local connectivity of the Julia set of the Feigenbaum polynomial.

\section{Renormalization AND tUning}

1.1. Quadratic-like mappings. To get more familiar with the notions of renormalization and polynomial-like mappings, one can for example consult $[\mathrm{DH}],[\mathrm{McM}]$ or $[\mathrm{S}]$.

Definition 1. Let us recall that a quadratic-like map $f: U^{\prime} \rightarrow U$ is a ramified covering of degree 2 between two topological disks $U^{\prime}$ and $U$, with $U^{\prime}$ relatively compact in $U$. One can define its filled-in Julia set $K(f)$ and its Julia set $J(f)$ as follows:

$$
\left\{\begin{array}{l}
K(f)=\left\{z \in U^{\prime} \mid(\forall n \in \mathbb{N}) f^{\circ n}(z) \in U^{\prime}\right\} \\
J(f)=\partial K(f) .
\end{array}\right.
$$

Examples. Let $P(z)=z^{2}+c$ be a quadratic polynomial. If $R$ is large enough, the restriction of $P$ to the disk $\mathbb{D}_{R}$ centered at 0 with radius $R$ is a quadratic-like map.

The restriction of the map $f(z)=2 \cos (z)-1.7+.9 i$ to the square

$$
U^{\prime}=\{z \mid-2<\operatorname{Re}(z)<2 \text { and }-2<\operatorname{Im}(z)<2\}
$$

is also a quadratic-like map. Figure 1 shows this quadratic-like map and its filled-in Julia set.

Let us also recall the so-called Straightening Theorem due to Douady and Hubbard. 


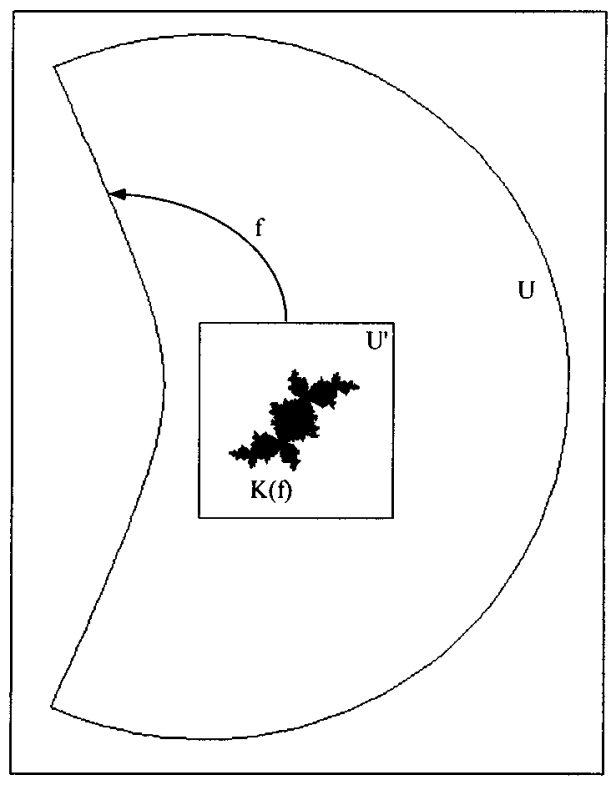

Figure 1. The quadratic-like map $z \rightarrow 2 \cos (z)-1.7+.9 i$ and its filled-in Julia set.

Theorem 1 (Straightening Theorem). If $f: U^{\prime} \rightarrow U$ is a quadratic-like map, then there exist

- a quadratic polynomial $P(z)=z^{2}+c$,

- a neighborhood $V$ of the filled-in Julia set $K(P)$ such that $P: P^{-1}(V)=$ $V^{\prime} \rightarrow V$ is a quadratic-like map, and

- a quasiconformal homeomorphism $\phi: U \rightarrow V$ with $\phi\left(U^{\prime}\right)=V^{\prime}$, such that $\bar{\partial} \phi=0$ almost everywhere on $K(f)$ and such that on $U^{\prime}$

$$
\phi \circ P=f \circ \phi .
$$

Moreover, if $K(f)$ is connected, then $P$ is unique.

Definition 2. Two quadratic-like maps $f$ and $g$ are said to be hybrid equivalent if there is a quasi-conformal conjugacy between them, with $\bar{\partial} h=0$ almost everywhere on the filled-in Julia set $K(f)$.

This defines an equivalence relation between quadratic-like mappings. The Straightening Theorem says that the hybrid class of a quadratic-like mapping $f$ always contains a quadratic polynomial, and that this polynomial is unique up to affine conjugacy if the Julia set $J(f)$ is connected.

1.2. Renormalization. Given a quadratic-like map $f: U^{\prime} \rightarrow U$ with a connected Julia set $J(f)$, there is always a fixed point $\alpha$ which is either non-repelling, or separating (i.e., $J(f) \backslash\{\alpha\}$ has several connected components). This point is called the $\alpha$ fixed point of $f$. The other fixed point is called the $\beta$ fixed point of $f$.

Definition 3. Let $f: U^{\prime} \rightarrow U$ be a quadratic-like map with connected Julia set, and let $\omega$ be its critical point. We say that $f$ is renormalizable with period $n>1$ 
if there exist an integer $n>1$, and open disks $V^{\prime} \subset V \subset \mathbb{C}$ containing $\omega$, such that

- the map $f^{\circ n}: V^{\prime} \rightarrow V$ is a quadratic-like map,

- its Julia set $J_{0}$ is connected, and

- the small Julia sets $J_{i}=f^{\circ i}\left(J_{0}\right), i=1, \ldots, n-1$, intersect $J_{0}$ at most at its $\beta$ fixed point.

The quadratic-like map $f^{\circ n}: V^{\prime} \rightarrow V$ is called a renormalization of $f$. Note that there are several possible choices of domains. Hence, there are several possible renormalizations of a given period. However, when the Julia set of the renormalization is connected, the problem can be solved by defining germs of quadratic-like maps. This will be done later.

Examples. Figure 2 shows the Julia set of the polynomial $P(z)=z^{2}-1.75$, together with the first three forward images of the disk $\Delta_{0}=\{z /|z|<.25\}$. The map $P^{\circ 3}: \Delta_{0} \rightarrow \Delta_{3}$ is a quadratic-like map, and its Julia set (which looks like a cauliflower) is connected. Hence, $P$ is renormalizable.

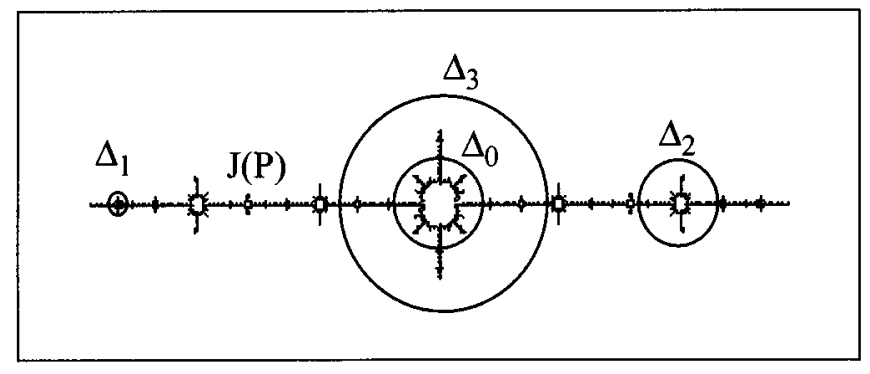

Figure 2. The polynomial $P(z)=z^{2}-1.75$ is renormalizable.

Now, given a quadratic polynomial $P$ which is renormalizable; we let $f=$ $P^{\circ n}: V^{\prime} \rightarrow V$ be a renormalization. One can construct a new quadratic polynomial by straightening the map $f$. The inverse process is called tuning.

1.3. Tuning. More precisely, given a parameter $c$ in the Mandelbrot set $M$, such that 0 is a periodic point of period $p$ for the polynomial $P_{c}(z)=z^{2}+c$, Douady and Hubbard $[\mathrm{DH}]$ have constructed a tuning map, $x \rightarrow c * x$, which is a homeomorphism of $M$ into itself, sending 0 to $c$, and such that if $x \neq 1 / 4$, then $f_{c * x}$ is renormalizable with period $p$, and the corresponding renormalization is in the same inner class as $P_{x}$. This is the way they show there are small copies of the Mandelbrot set within itself (see Figure 3).

The central object in our study is the Feigenbaum polynomial. One can define this polynomial as the unique real polynomial which is a fixed point of tuning by -1 . In other words, the Feigenbaum parameter $c_{F e i g}=-1.401155 \ldots$, is the unique real parameter in the intersection of all the copies of $M$ obtained by tuning by -1 . This intersection is not known to consist of a single point, but its real trace is known to be reduced to a single point. Figure 4 shows the location of the parameter $c_{F e i g}$ in the Mandelbrot set, and Figure 5 shows the Julia set of the Feigenbaum polynomial.

The Feigenbaum polynomial is the most famous example of a polynomial to be infinitely renormalizable. By construction, the Feigenbaum polynomial, $P_{F e i g}$, is 


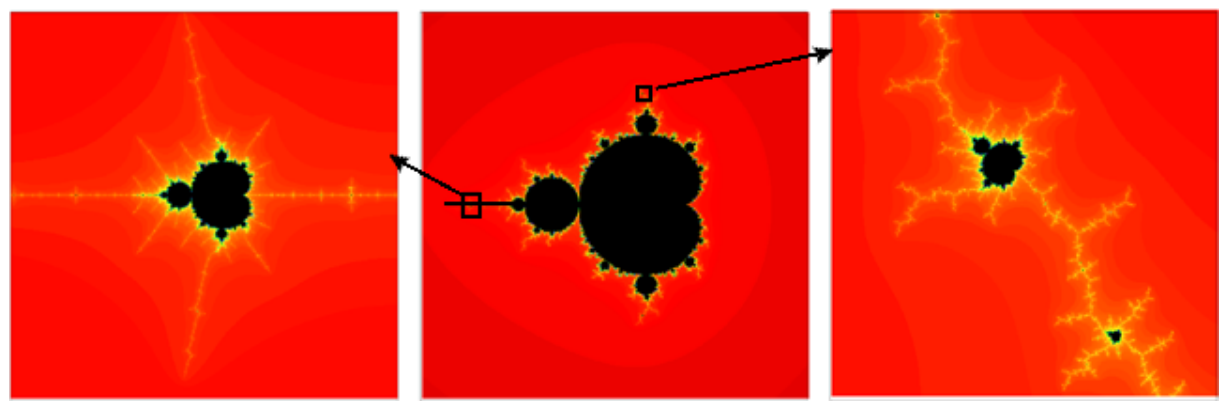

Figure 3. The Mandelbrot set and two small copies corresponding to renormalization of period 3 .
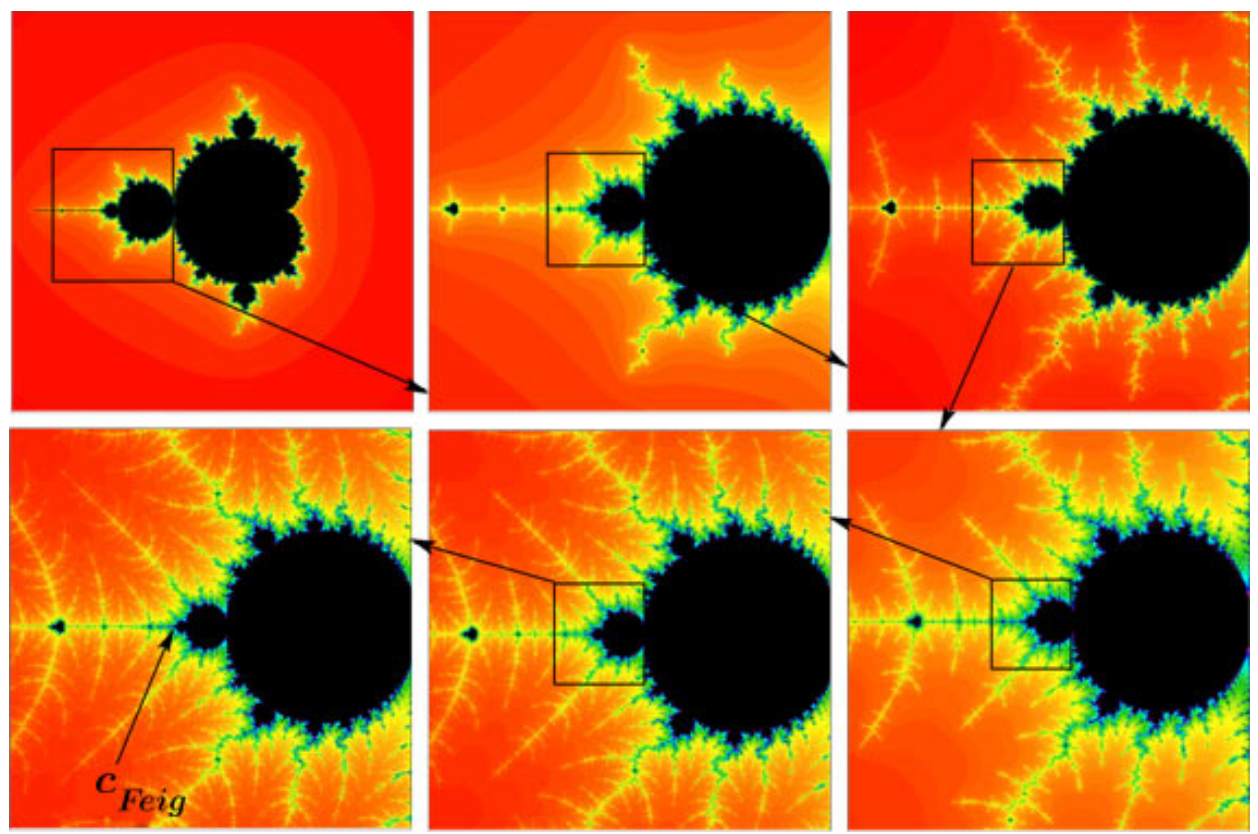

FiguRE 4 . The location of the parameter $c_{F e i g}$ in the Mandelbrot set.

renormalizable with period 2, i.e., there exist domains $U^{\prime}$ and $U$ such that $f=$ $P^{\circ 2}: U^{\prime} \rightarrow U$ is a quadratic-like mapping with connected Julia set. There are several possible choices of domains, hence several renormalizations. However, all have the same Julia set, and are equal on this Julia set.

Definition 4. We will say that two quadratic-like maps $f: U^{\prime} \rightarrow U$ and $g: V^{\prime} \rightarrow$ $V$ with connected Julia set define the same germ $[f]$ of quadratic-like map if $J(f)=$ $J(g)$ and $f|J(f)=g| J(g)$.

We can define a renormalization operator $\mathcal{R}_{2}$ in the following way.

Definition 5. Assume $[f]$ is a germ of a quadratic-like map which is renormalizable with period 2. There exist open sets $U^{\prime}$ and $U$ such that the map $g: U^{\prime} \rightarrow U$ defined by $g=f^{\circ 2} \mid U$ is a polynomial-like map with connected Julia set. The 


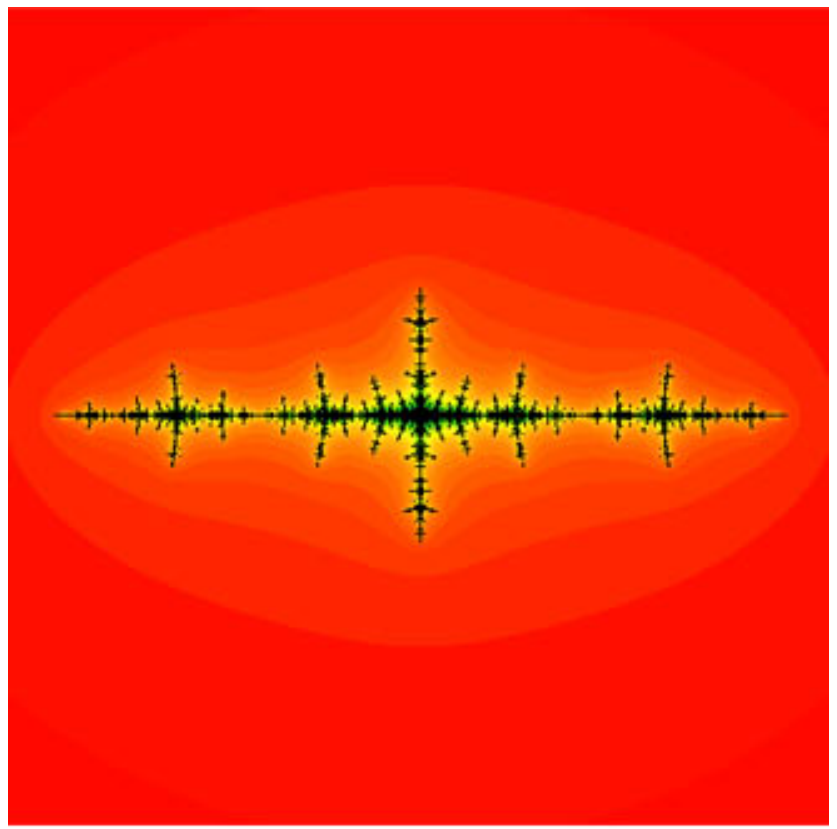

Figure 5. The Julia set of the Feigenbaum polynomial.

renormalization operator $\mathcal{R}_{2}$ is defined by

$$
\mathcal{R}_{2}([f])=\left[\alpha^{-1} \circ g \circ \alpha\right],
$$

with $\alpha=g(0)=f^{2}(0)$, and $\alpha(z)=\alpha z$.

We have normalized the germ so that the critical value is 1 .

Definition 6. Two quadratic-like germs $[f]$ and $[g]$ are said to be hybrid equivalent if there exist representatives $f: U^{\prime} \rightarrow U$ and $g: V^{\prime} \rightarrow V$ which are hybrid equivalent.

The equivalence classes are called hybrid classes of germs.

Now, let $P_{F e i g}$ be the Feigenbaum polynomial. The Straightening Theorem says that there is a unique polynomial $Q(z)=z^{2}+c$ in the hybrid class of $\mathcal{R}_{2}\left(\left[P_{\text {Feig }}\right]\right)$. This polynomial must be renormalizable with period $2^{k}$, for all $k \geq 1$. Besides, it must be a real polynomial. So it is the Feigenbaum polynomial. Hence, we can define a sequence of germs $\mathcal{R}_{2}^{\circ n}\left(\left[P_{\text {Feig }}\right]\right), n \in \mathbb{N}$. The following result can be found in $[\mathrm{S}],[\mathrm{dMvS}]$ or $[\mathrm{McM}]$.

Theorem 2. The sequence of germs $\mathcal{R}_{2}^{\circ n}\left(\left[P_{\text {Feig }}\right]\right), n \in \mathbb{N}$, converges to a point $[f]$. This germ is a fixed point of renormalization:

$$
\mathcal{R}_{2}([f])=[f],
$$

and is in the hybrid class of the Feigenbaum polynomial. It is the unique fixed point of $\mathcal{R}_{2}$. 


\section{The Cvitanović-Feigenbaum equation}

We will first state some results about this fixed point of renormalization. It is just a germ of quadratic-like map. However, Epstein [E2] proved that there exists a natural representative of this germ. We think it is worth making the construction here, to help the reader get accustomed with the tools.

First of all, we would like to recall that the fixed point $[f]$ of $\mathcal{R}_{2}$ is a solution of the following system of equations (see [E2]).

Definition 7. The Cvitanović-Feigenbaum equation:

$$
\left\{\begin{array}{l}
f(z)=-\frac{1}{\lambda} f \circ f(\lambda z) \\
f(0)=1 \\
\left.f(z)=F\left(z^{2}\right), \text { with } F^{-1} \text { univalent in } \mathbb{C} \backslash(]-\infty,-1 / \lambda\right] \cup\left[1 / \lambda^{2},+\infty[) .\right.
\end{array}\right.
$$

Theorem 3 (Epstein, see [E2]). Let $f$ be a solution of the equation, and let $x_{0}$ be the first positive preimage of 0 by $f$. Then,

- $f(1)=-\lambda$,

- $f\left(\lambda x_{0}\right)=x_{0}$, and

- the first critical point in $\mathbb{R}^{+}$is $x_{0} / \lambda$, with $f\left(x_{0} / \lambda\right)=-1 / \lambda$.

Figure 6 illustrates Proposition 3. This graph gives the relative positions of some points on the real axis.

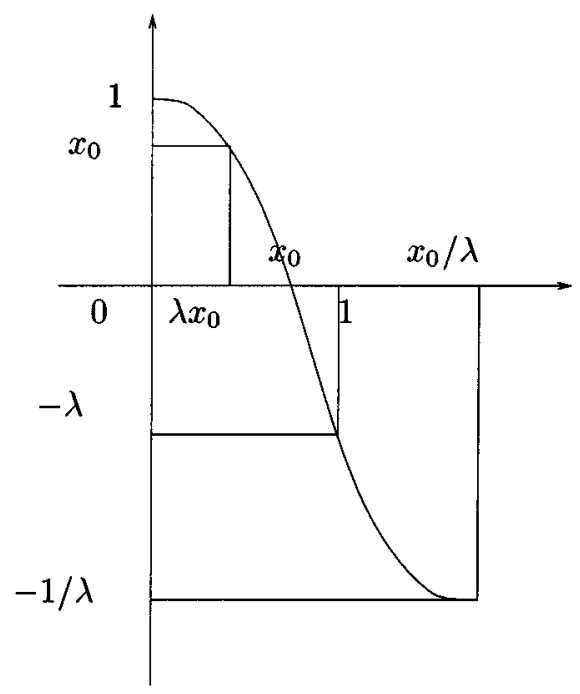

Figure 6 . The graph of $f$ on $\mathbb{R}^{+}$.

Theorem 4 (Epstein, see [E2]). Let $F$ be defined by the Cvitanović-Feigenbaum equation. We then have the following results:

- it is possible to extend $F^{-1}$ continuously to the boundary $\mathbb{R}$ of $\mathbb{H}_{+}$, and even analytically except at points $(-1 / \lambda)^{n}, n \geq 1$, which are branching points of type $z^{1 / 2}$,

- the values of $F^{-1}$ are never real except in $\left[-1 / \lambda, 1 / \lambda^{2}\right]$,

- the extension of $F^{-1}$ to the closure of $\mathbb{H}_{+}$is injective, and

- when z tends to infinity in $\mathbb{H}_{+}, F^{-1}(z)$ tends to a point in $\mathbb{H}_{-}$which will be denoted by $F^{-1}(i \infty)$. 
By symmetry, similar statements hold in $\mathbb{H}_{-}$. Hence, $\mathcal{W}=F^{-1}\left(\mathbb{C}_{\lambda}\right)$ is a bounded domain of $\mathbb{C}$. Those results are summarized in Figure 7.

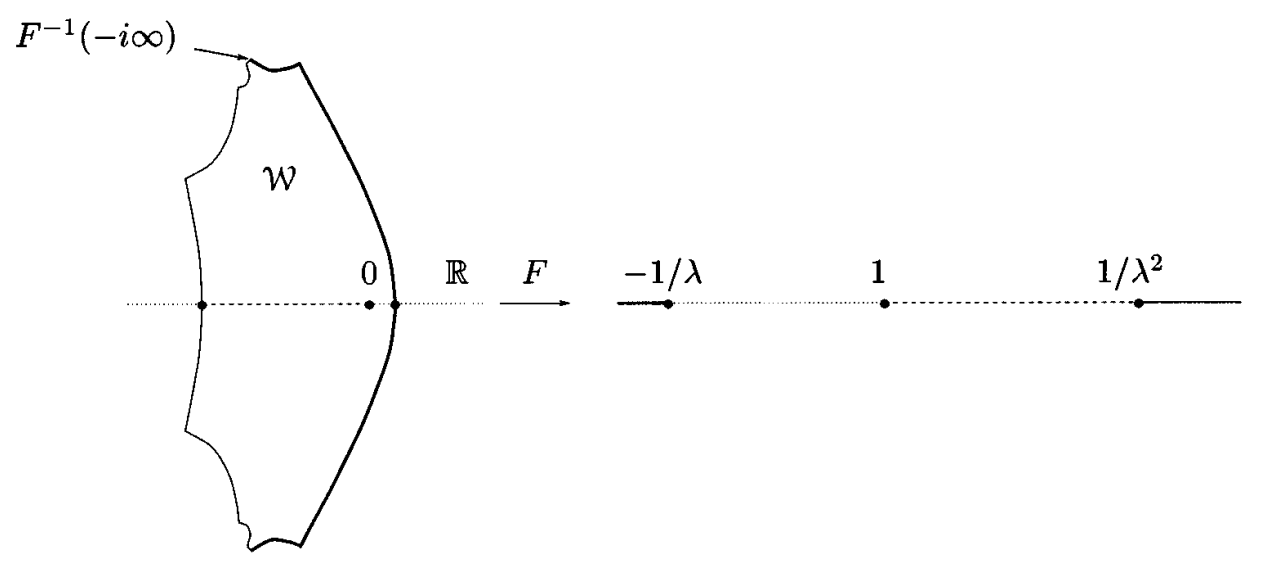

FiguRE 7. Maximal univalent extension of $F$.

In the following, we will use the notations:

$$
\begin{aligned}
\mathbb{C}_{\lambda} & =\mathbb{C} \backslash(]-\infty,-1 / \lambda] \cup\left[1 / \lambda^{2},+\infty[),\right. \\
\mathcal{W} & =F^{-1}\left(\mathbb{C}_{\lambda}\right), \\
W & =\left\{z \in \mathbb{C} \mid z^{2} \in \mathcal{W}\right\}, \\
W_{+} & =W \cap\{z \in \mathbb{C} \mid 0<\operatorname{Arg}(z)<\pi / 2\}, \text { and } \\
W_{-} & =W \cap\{z \in \mathbb{C} \mid-\pi / 2<\operatorname{Arg}(z)<0\} .
\end{aligned}
$$

As $\bar{W}_{+} \cap \mathbb{R}=\left[0, x_{0} / \lambda\right]$, we can deduce that $\left.W \cap \mathbb{R}=\right]-x_{0} / \lambda, x_{0} / \lambda[$.

Corollary 1. The map $f: W \rightarrow \mathbb{C}_{\lambda}$ is a quadratic-like map representing the renormalization fixed point.

Proof. The graph of $f$ (see Figure 6) makes it possible to conclude that $\bar{W} \subset \mathbb{C}_{\lambda}$, because $x_{0}<1$. Hence $f: W \rightarrow \mathbb{C}_{\lambda}$ is a quadratic-like map. Besides, $f$ has a unique critical point at 0 . The corollary follows from the uniqueness of the fixed point.

This quadratic-like map is in the hybrid class of the Feigenbaum polynomial. Thus, to obtain informations like local connectivity, Hausdorff dimension or Lebesgue measure of the Julia set of the Feigenbaum polynomial, it is enough to know the corresponding results for the Julia set $K(f)$ of this quadratic-like map (see Figure 8).

Definition 8. In the following, we will use the notation

$$
W_{n}=f^{-2^{n}}\left(\mathbb{C}_{\lambda}\right) .
$$

Observe that since $f: W \rightarrow \mathbb{C}_{\lambda}$ is a polynomial-like map with non-escaping critical point, the set $W_{n}$ is simply connected, $W_{n+1} \subset W_{n}$, and $K(f)=\bigcap W_{n}$. 


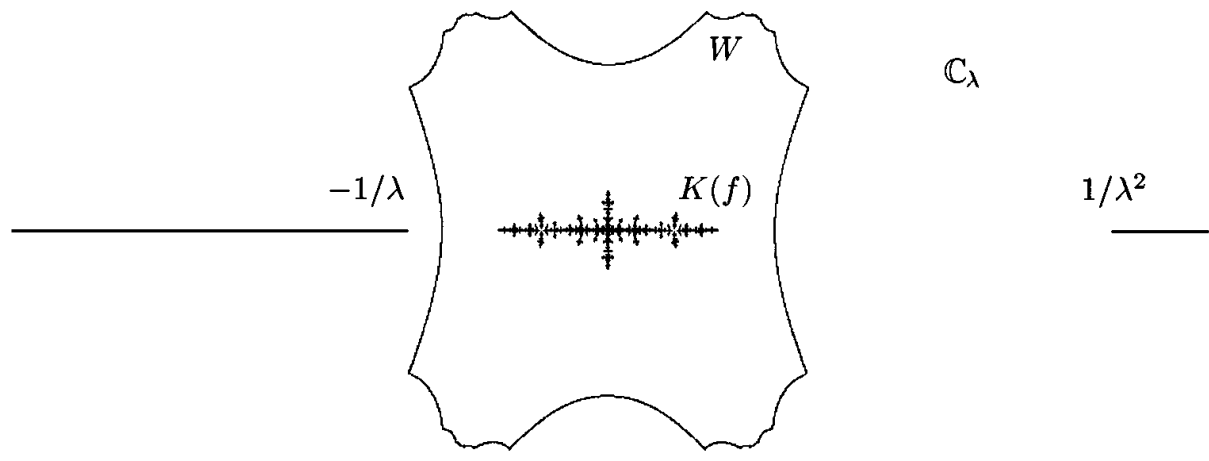

Figure 8. A representative of the Feigenbaum fixed point of renormalization, and the Julia set $K(f)$.

\section{Geometry of the domain of analyticity of $f$}

Our first goal is to describe the geometry of the maximal domain of analyticity $\widehat{W}$ of $f$. Epstein [E2] proved that it is an open simply connected subset of $\mathbb{C}$ and McMullen $[\mathrm{McM}]$ (7.3) proved that this domain is a dense subset of $\mathbb{C}$. We will show that $\widehat{W}$ is contained in $\widehat{W} / \lambda$, and that it can be seen as the basin of attraction of a map related to $f$. We will then introduce a tiling and some puzzles. We will use them to give a combinatorial description of $f$ and to prove the local connectivity of the Julia set $K(f)$ at the critical point.

\subsection{The domain of analyticity.}

Definition 9. Let $f$ and $g$ be two holomorphic functions defined on open connected domains of $\mathbb{C}: U_{f}$ and $U_{g}$. We say that $g$ is an analytic extension of $f$ if $f=g$ on some non-empty open set. Moreover, if all such analytic extensions are restrictions of a single map

$$
\hat{f}: \widehat{W} \rightarrow \mathbb{C},
$$

we will say that $\hat{f}$ is the maximal analytic extension of $f$.

Proposition 1 (see [E2]). Let $f$ be a solution of the Cvitanović-Feigenbaum equation. It has a maximal analytic extension

$$
\hat{f}: \widehat{W} \rightarrow \mathbb{C},
$$

where $\widehat{W}$ is a simply connected open subset of $\mathbb{C}$.

Proof. First of all, recall that $f: W \rightarrow \mathbb{C}_{\lambda}$ is a quadratic-like map with nonescaping critical point. Hence, the set $W_{n}=f^{-2^{n}}\left(\mathbb{C}_{\lambda}\right)$ is a simply connected open set and the map $f^{\circ 2^{n}}: W_{n} \rightarrow \mathbb{C}_{\lambda}$ is a proper map. But the Cvitanović-Feigenbaum equation shows that

$$
f_{n}=\left(-\frac{1}{\lambda}\right)^{n} f^{\circ 2^{n}}\left(\lambda^{n} z\right): \frac{1}{\lambda^{n}} W_{n} \rightarrow \frac{1}{(-\lambda)^{n}} \mathbb{C}_{\lambda}
$$

is an extension of $f$. 
Moreover, as

$$
\frac{1}{(-\lambda)^{n-1}} \mathbb{C}_{\lambda} \subset \frac{1}{(-\lambda)^{n}} \mathbb{C}_{\lambda},
$$

the range of $f_{n-1}$ is contained in the range of $f_{n}$. Since the domains are connected, we claim that the inclusion also holds for the domains:

$$
\frac{1}{\lambda^{n-1}} W_{n-1} \subset \frac{1}{\lambda^{n}} W_{n} .
$$

Indeed, consider the connected component $U$ of $W_{n-1} / \lambda^{n-1} \cap W_{n} / \lambda^{n}$ which contains 0 . The two mappings $f_{n-1}$ and $f_{n}$ coincide on this component. Besides, if the inclusion of the domains does not hold, $U$ must have a boundary point in $W_{n-1} / \lambda^{n-1}$, which is mapped by $f_{n-1}$ in the interior of $\mathbb{C}_{\lambda} /(-\lambda)^{n}$. This contradicts the properness of $f_{n}$.

We can now define

$$
\widehat{W}=\bigcup_{n \in \mathbb{N}} \frac{1}{\lambda^{n}} W_{n}
$$

The map $f$ has an analytic extension $\hat{f}$ defined on $\widehat{W}$ which coincides with $f_{n}$, if $z \in W_{n} / \lambda^{n}$.

Next, let us prove that we cannot extend $\hat{f}$ across any point of $\partial \widehat{W}$. Any point $z \in \partial \widehat{W}$ can be approximated by points $z_{n} \in \partial\left(W_{n} / \lambda^{n}\right)$. On the other hand, $\left|\hat{f}\left(z_{n}\right)\right|=\left|f_{n}\left(z_{n}\right)\right|$ tends to infinity when $n$ tends to infinity. Besides, $z$ cannot be a pole since there is no isolated point in the boundary of $\widehat{W}$. Hence, we cannot extend $\hat{f}$ across any point of $\partial \widehat{W}$. This shows that $\hat{f}$ is a maximal analytic extension of $f$.

To lighten the notations, we will denote by $f$ - instead of $\hat{f}$ - the maximal analytic extension, when there is no ambiguity. However, $K(f)$ still denotes the Julia set of the quadratic-like restriction $f: W \rightarrow \mathbb{C}_{\lambda}$.

\section{ThE MAP $f$ IS A LINEARIZER}

This section is devoted to the proof of the following result.

Theorem A. The map $f_{\lambda}(z)=f(\lambda z)$ defined on $\widehat{W} / \lambda$ has a fixed point $x_{0} \in \widehat{W}$ with multiplier $-\lambda$. Besides, $f_{\lambda}$ is analytic on $\widehat{W}, f_{\lambda}(\widehat{W})=\widehat{W}$, and $\widehat{W}$ is the basin of attraction of the map $f_{\lambda}$. Finally, $f$ is a linearizer of the map $f_{\lambda}$.

Proof. Let us first prove that the map $f_{\lambda}$ has an attracting fixed point. In fact, we will prove a little bit more for later purposes.

Lemma 1. The point $x_{0}$ is an attracting fixed point of the map $f_{\lambda}(z)=f(\lambda z)$. Its multiplier is $-\lambda$. Moreover $f_{\lambda}: W_{+} \rightarrow W_{-}$and $f_{\lambda}: W_{-} \rightarrow W_{+}$are isomorphisms.

Remark. The fact that $f_{\lambda}$ is an isomorphism from $W_{+}$onto $W_{-}$does not mean that $f_{\lambda}$ is an automorphism of $W_{+} \cup W_{-}$. Indeed, part of the boundary of $W_{ \pm}$is mapped inside $W_{+} \cup W_{-}$. Hence $f_{\lambda}$ maps $W_{+} \cup W_{-}$onto $W_{+} \cup W_{-}$minus a slit. This is the way $f_{\lambda}$ can have an attracting fixed point $x_{0}$ inside $W_{+} \cup W_{-}$. 
Proof. The trick in the proof is that the Cvitanović-Feigenbaum equation can be translated on the following commutative diagram:

$$
\begin{array}{rll}
\left(\widehat{W}, x_{0}\right) & \stackrel{f_{\lambda}}{\longrightarrow} & \left(\widehat{W}, x_{0}\right) \\
f \downarrow & & \downarrow f \\
(\mathbb{C}, 0) & \underset{z \mapsto-\lambda z}{\longrightarrow} & (\mathbb{C}, 0) .
\end{array}
$$

It is a linearizing equation which proves the first part of the proposition.

Next, in Proposition 1, we obtained the following inclusions:

$$
\lambda W \subset W_{1} \subset W .
$$

Hence, $\lambda W_{+} \subset W_{+}$, and since $f: W_{+} \rightarrow \mathbb{H}_{-}$is an isomorphism, we see that $f_{\lambda}$ is an isomorphism from $W_{+}$onto its image. On the other hand,

$$
\mathbb{H}_{-}=f\left(W_{+}\right)=-\frac{1}{\lambda} f \circ f\left(\lambda W_{+}\right) .
$$

Hence $f\left(f_{\lambda}\left(W_{+}\right)\right)=\mathbb{H}_{+}$, and the set $f_{\lambda}\left(W_{+}\right)$is a preimage of $\mathbb{H}_{+}$under $f$. As $x_{0}$ is in the closure of $W_{+}, x_{0}=f_{\lambda}\left(x_{0}\right)$ is in the closure of $f_{\lambda}\left(W_{+}\right)$. Hence, we can immediately deduce that $f_{\lambda}\left(W_{+}\right)=W_{-}$. We can use the same arguments to show that $f_{\lambda}: W_{-} \rightarrow W_{+}$is an isomorphism.

Next, let us show that the map $f_{\lambda}$ is analytic on $\widehat{W}$. We have to show that $\lambda \widehat{W}$ is contained in the domain of analyticity of $f$.

Lemma 2. The domain $\lambda \widehat{W}$ is contained in $\widehat{W}$ and $f_{\lambda}(\widehat{W}) \subset \widehat{W}$.

Proof. By construction, $W_{n+1} \subset W_{n}$, and in the proof of Proposition 1, we saw that $W_{n} / \lambda^{n} \subset W_{n+1} / \lambda^{n+1}$. Hence,

$$
\lambda \frac{1}{\lambda^{n}} W_{n} \subset \lambda \frac{1}{\lambda^{n+1}} W_{n+1} \subset \frac{1}{\lambda^{n}} W_{n} .
$$

Since the sets $W_{n} / \lambda^{n}$ give an exhaustion of $\widehat{W}$, this proves that $\lambda \widehat{W} \subset \widehat{W}$. In particular, $f$ is defined on $\lambda \widehat{W}$.

Now, notice that $f^{\circ 2^{n}}\left(W_{n+1}\right)=W_{n}$. Hence,

$$
f\left(\lambda \frac{1}{\lambda^{n}} W_{n}\right) \subset f\left(\lambda \frac{1}{\lambda^{n+1}} W_{n+1}\right)=\left(-\frac{1}{\lambda}\right)^{n} f^{\circ 2^{n}}\left(W_{n+1}\right)=\frac{1}{\lambda^{n}} W_{n} .
$$

This shows that $f(\lambda \widehat{W}) \subset \widehat{W}$.

We will now finish the proof of Theorem A. We will prove that $\widehat{W}$ is that immediate basin of attraction of the map $f_{\lambda}$. Since McMullen proved that $\widehat{W}$ is dense in $\mathbb{C}$, there cannot be any other component in the basin of $f_{\lambda}$.

The commutative diagram we have written tells us that the linearizer of the map $f_{\lambda}$ is $f$. We claim that the domain of analyticity of the linearizer is the immediate basin of the attracting fixed point $x_{0}$.

To avoid confusion let us use the name $\phi$ for the linearizer. We can always extend the linearizer to the immediate basin of attraction using the formula:

$$
\phi(z)=\frac{1}{\lambda^{n}} f_{\lambda}^{\circ n}(z)
$$

where $n$ is so chosen large enough so that $f_{\lambda}^{\circ n}(z)$ is in a neighborhood of $x_{0}$ where $\phi$ is already defined. This proves that the immediate basin of attraction is contained in $\widehat{W}$. 
To show the reverse inclusion, we just need to use Lemma 2: the mapping $f_{\lambda}$ is analytic on $\widehat{W}$ and maps this domain into itself. Since $\widehat{W}$ is connected, Schwartz's Lemma shows that $f_{\lambda}$ is contracting on $\widehat{W}$ for its Poincaré metric. Hence $\widehat{W}$ is contained in the immediate basin of attraction of $f_{\lambda}$.

The following two propositions are immediate applications of this result.

Proposition 2. We have the following inclusion of sets:

$$
\lambda K(f) \subset K(f) \subset \bigcup_{n \in \mathbb{N}} \frac{K(f)}{\lambda^{n}} \subset \bigcap_{n \in \mathbb{N}} \lambda^{n} \widehat{W} \subset \widehat{W} \subset \frac{\widehat{W}}{\lambda} .
$$

Proof. The set $\lambda K(f)$ is the Julia set $K\left(f^{\circ 2}\right)$ of the renormalization

$$
f^{\circ 2}: \lambda W \rightarrow \lambda \mathbb{C}_{\lambda} .
$$

This Julia set is contained in $K(f)$. The inclusion

$$
K(f) \subset \bigcup_{n \in \mathbb{N}} K(f) / \lambda^{n}
$$

follows immediately.

The inclusion $\widehat{W} \subset \widehat{W} / \lambda$ is given by Lemma 2 . The inclusion

$$
\bigcap_{n \in \mathbb{N}} \lambda^{n} \widehat{W} \subset \widehat{W}
$$

follows immediately from this one.

Lemma 3. The sets $K(f) / \lambda^{n},(n \in \mathbb{N})$, are all contained in $\widehat{W}$.

Proof. The domain $\widehat{W}$ is the union of the sets $W_{n} / \lambda^{n}$. But $K(f) \subset W_{n}=$ $f^{-2^{n}}\left(\mathbb{C}_{\lambda}\right)$. Hence, for all $n \in \mathbb{N}$,

$$
K(f) / \lambda^{n} \subset W_{n} / \lambda^{n} \subset \widehat{W}
$$

The remaining inclusion

$$
\bigcup_{n \in \mathbb{N}} \frac{K(f)}{\lambda^{n}} \subset \bigcap_{n \in \mathbb{N}} \lambda^{n} \widehat{W}
$$

follows from this lemma.

McMullen proved that the union $\bigcup_{n \in \mathbb{N}} K(f) / \lambda^{n}$ is dense in $\mathbb{C}$. Hence $\widehat{W}$ is a dense open subset of $\mathbb{C}$. As $K(f)$ is a compact set with empty interior, the inclusions are strict because of Baire's Theorem: a countable union of closed sets with empty interior cannot be equal to a countable intersection of dense open sets.

We have used this information to draw the boundary of $\widehat{W}$. Figure 9 shows a blow-up near the critical point of the Feigenbaum Julia set. The points which are colored in black correspond to points in the boundary of $\widehat{W}$. In the next section, we will describe a tiling of the domain $\widehat{W}$. We would like to mention that Epstein has a very nice picture of the domain of analyticity $\widehat{W}$ (see [E2]).

Finally, the intersection of the contractions $\lambda^{n} \widehat{W}$ can be defined dynamically. 


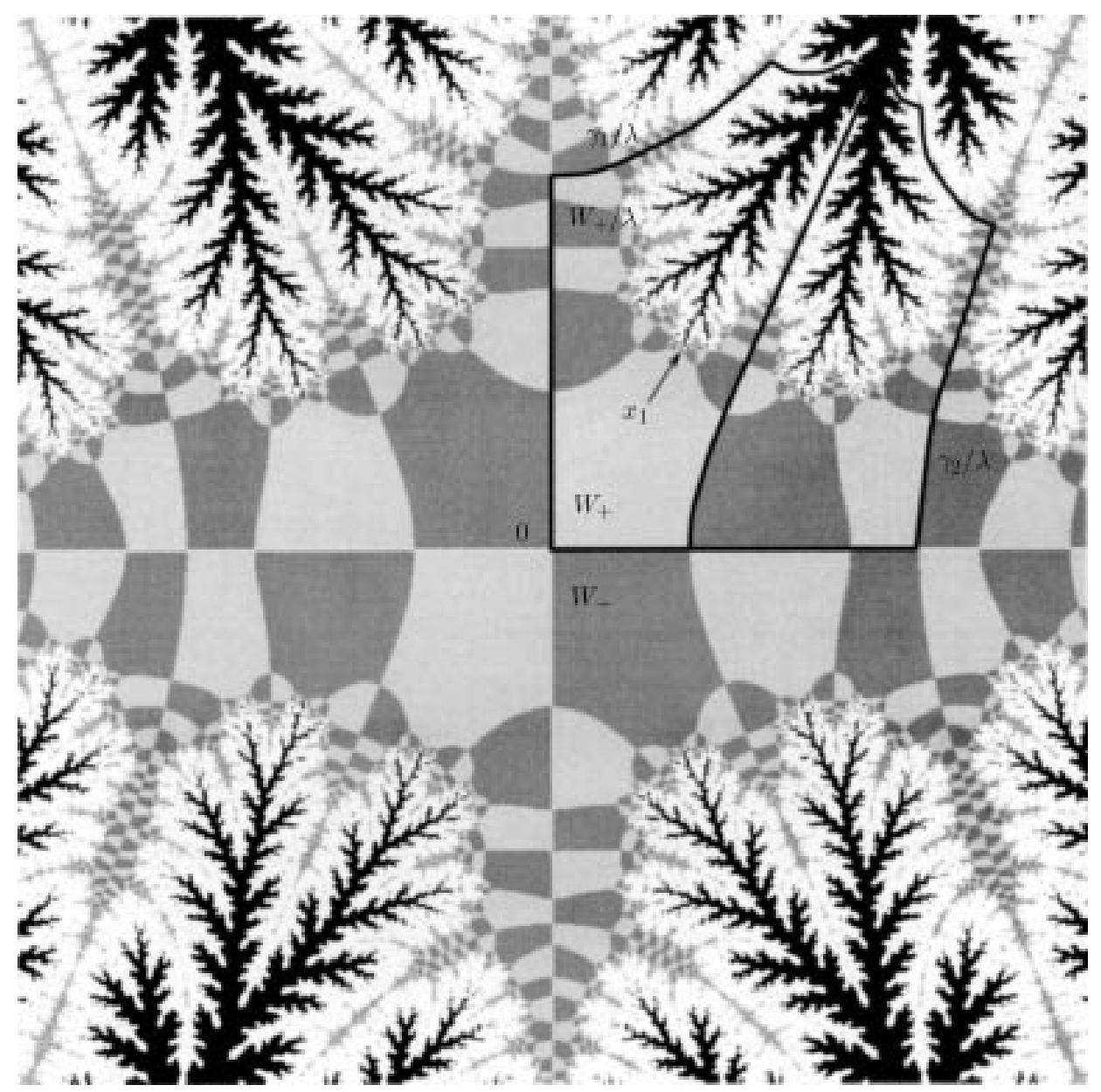

Figure 9. The domain of analyticity of the Feigenbaum fixed point of renormalization. Everything which is not black, is in the domain of analyticity. The map $f$ sends light tiles to $\mathbb{H}_{-}$and dark tiles to $\mathbb{H}_{+}$.

Proposition 3. The intersection of all the sets $\lambda^{n} \widehat{W}$ is the set of points whose orbit under iteration of $f: \widehat{W} \rightarrow \mathbb{C}$ stay in $\widehat{W}$ :

$$
\bigcap_{n \in \mathbb{N}} \lambda^{n} \widehat{W}=\widehat{K}=\left\{z \in \widehat{W} \mid(\forall n \in \mathbb{N}) f^{\circ n}(z) \in \widehat{W}\right\} .
$$

Proof. To show this equality, note that

$$
\widehat{K}=\bigcap_{n \in \mathbb{N}} f^{-n}(\widehat{W}) .
$$

We have noticed that $f(\lambda \widehat{W})=f_{\lambda}(\widehat{W})=\widehat{W}$. Moreover, in a neighborhood of the origin,

$$
f(z)=-\frac{1}{\lambda} f \circ f(\lambda z)
$$


This equality has to be true whenever both sides are simultaneously defined. The left-hand side is defined on $\widehat{W}$. When $z \in \widehat{W}, f_{\lambda}(z) \in \widehat{W}$, and the right-hand side of the equality is defined. The Cvitanović-Feigenbaum equation holds on the whole domain $\widehat{W}$.

As $f^{-1}(\widehat{W})=\lambda \widehat{W}$, it follows by induction on $n$ that $f^{-\left(2^{n}-1\right)}(\widehat{W})=\lambda^{n} \widehat{W}$. Indeed, the Cvitanović-Feigenbaum equation shows that

$$
f^{-1}(z)=\frac{1}{\lambda^{n}} f^{-2^{n}}\left(\lambda^{n} z\right)
$$

Hence,

$$
f^{-2^{n}}\left(\lambda^{n} \widehat{W}\right)=\lambda^{n} f^{-1}(\widehat{W})=\lambda^{n+1} \widehat{W} .
$$

Replacing $\lambda^{n} \widehat{W}$ by $f^{-\left(2^{n}-1\right)}(\widehat{W})$ shows that the induction property holds for $n+1$. The Theorem follows immediately from this observation.

\section{Combinatorial description of the map $f: \widehat{W} \rightarrow \mathbb{C}$}

\subsection{The map $f: \widehat{W} \rightarrow \mathbb{C}$ is a ramified covering.}

Theorem B. All the critical points of $f$ are simple. The critical values of $f$ are all contained in the real axis. Besides, the analytic extension $f: \widehat{W} \rightarrow \mathbb{C}$ is a ramified covering, in the sense that for any bounded disk $\mathbb{D}_{R} \subset \mathbb{C}$, each component of $f^{-1}\left(\mathbb{D}_{R}\right)$ is proper over $\mathbb{D}_{R}$.

Proof. Remember that on $W_{n} / \lambda_{n}$, the map $f$ coincides with

$$
\left(-\frac{1}{\lambda}\right)^{n} f^{\circ 2^{n}}\left(\lambda^{n} z\right)
$$

The critical points of $f^{\circ 2^{n}}$ in $W_{n}$ are preimages of 0 by the polynomial-like map $f: W \rightarrow \mathbb{C}_{\lambda}$. Those are all simple critical points. By rescaling with a factor $(-1 / \lambda)^{n}$ we see that the critical points of $f$ in $W_{n} / \lambda_{n}$ are simple. Since $\widehat{W}$ is the union of the sets $W_{n} / \lambda_{n}$, the statement for critical points follows.

The critical orbit of the polynomial-like map $f: W \rightarrow \mathbb{C}_{\lambda}$ is contained in the real axis. Hence, rescaling with a factor $(-1 / \lambda)^{n}$, we see that the critical values of $f$ are all contained in the real axis.

To finish the proof of Theorem B, we need the following lemma.

Lemma 4. If $U \subset \widehat{W}$ is not relatively compact in $\widehat{W}$, then $f(U)$ is not relatively compact in $\mathbb{C}$.

Remark. This lemma implies for example that $f$ has no singular value in $\mathbb{C}$.

Proof. Remember that the set $W_{n}$ has been defined via the quadratic-like map $f: W \rightarrow \mathbb{C}_{\lambda}$ as

$$
W_{n}=\left\{z \in W \mid f^{\circ 2^{n}}(z) \in \mathbb{C}_{\lambda}\right\}
$$

in particular, $W_{n}$ is relatively compact in $W_{n-1}$. Hence, $W_{n} / \lambda^{n}$ is relatively compact in the domain of analyticity of $f_{\lambda}: \widehat{W} / \lambda$. Besides,

$$
f_{\lambda}\left(\frac{1}{\lambda^{n+1}} W_{n+1}\right)=\left(-\frac{1}{\lambda}\right)^{n} f^{\circ 2^{n}}\left(W_{n+1}\right)=\frac{1}{\lambda^{n}} W_{n} .
$$

Thus, $f_{\lambda}^{\circ n}$ is analytic on a neighborhood of $W_{n} / \lambda_{n}$, and maps $W_{n} / \lambda_{n}$ to $W$. 
Now, if $U \subset \widehat{W}$ is not relatively compact in $\widehat{W}$, we have only two possibilities:

- either $U$ is entirely contained in one of the sets $W_{n} / \lambda^{n}$; in that case $\bar{U}$ contains a point in $\overline{W_{n} / \lambda^{n}} \cap \partial \widehat{W}$. Those points are mapped by $f_{\lambda}^{\circ n}$ to $x_{1},-x_{1}, \bar{x}_{1}$ or $-\bar{x}_{1}$. Hence $f_{\lambda}^{\circ n}(\bar{U})$ is contained in $\bar{W}$ and contains one of those points, let's say $x_{1}$. Since $f(z)$ tends to infinity when $z \in W$ tends to $x_{1}$, and since

$$
f(U)=\left(-\frac{1}{\lambda}\right)^{n} f\left(f_{\lambda}^{\circ n}(U)\right),
$$

we see that $U$ cannot be compact in $\mathbb{C}$;

- or $U$ intersects the boundary of infinitely many sets $W_{n} / \lambda^{n}$; in that case, its image has to intersect the boundary of infinitely many sets $\mathbb{C}_{\lambda} / \lambda^{n}$ and is not compact in $\mathbb{C}$.

We now come back to the proof of Theorem B. The critical points of $f$ are discrete in $\widehat{W}$ (since otherwise $f^{\prime}$ would be identically 0 ). Hence, to see that $f$ is a ramified cover, it is enough to show that for any disk $\mathbb{D}_{R} \subset \mathbb{C}$ centered at 0 with radius $R$, the connected components of $f^{-1}\left(\mathbb{D}_{R}\right)$ are relatively compact in $\widehat{W}$.

So, we let $V \subset \widehat{W}$ be a neighborhood of $x_{0}$ such that $f: V \rightarrow \mathbb{C}$ is an isomorphism onto the disk centered at 0 of radius $r$, and we suppose that $f^{-1}\left(\mathbb{D}_{R}\right)$ has a connected component $U$ which is not relatively compact in $\widehat{W}$. By Lemma 4 , the sets $f_{\lambda}^{\circ n}(U)$ are never relatively compact in $\widehat{W}$. Indeed,

- either $U$ is relatively compact in the domain $\widehat{W} / \lambda$ of $f_{\lambda}$; in that case, $\bar{U}$ contains a point of the boundary of the basin, $f_{\lambda}(\bar{U})$ also since $f_{\lambda}$ is analytic on a neighborhood of $\bar{U}$,

- or $U$ is not relatively compact in $\widehat{W} / \lambda$ and $f_{\lambda}(U)$ is not compact in $\mathbb{C}$.

Besides, since every point in $U$ is attracted by $x_{0} \in V$, there exists an integer $n_{0}$ such that $f_{\lambda}^{\circ n_{0}}(W) \cap V \neq \emptyset$. Since $f_{\lambda}(V) \subset V$, for every $n \geq n_{0}, f_{\lambda}^{\circ n}(W) \cap V \neq \emptyset$, and since $W$ is connected there exists a point $w_{n} \in W$ such that $f_{\lambda}^{\circ n}\left(w_{n}\right) \in \partial V$. Hence,

$$
R \geq\left|f\left(w_{n}\right)\right|=\left|\frac{f\left(f_{\lambda}^{\circ n}\left(w_{n}\right)\right)}{(-\lambda)^{n}}\right|=\frac{r}{\lambda^{n}},
$$

which gives a contradiction if $n$ is large enough so that $R<r / \lambda^{n}$.

5.2. Defining a tiling of the domain of analyticity. Given a point $x \in \widehat{W}$, the orbit of $x$ under $f_{\lambda}$ is attracted by the fixed point $x_{0}$. Hence, either the orbit is eventually contained in the real axis, or the orbit eventually enter the pieces $W_{ \pm}$.

Definition 10. For any $x \in \widehat{W}$, if $f_{\lambda}^{\circ n}(x) \in W_{ \pm}$, then the puzzle piece $P_{x}$ around $x$ is the connected component of $f_{\lambda}^{-n}\left(W_{ \pm}\right)$that contains $x$. We will say that $p$ is a tile of $\widehat{W}$ if and only if $p=\bar{P}_{x} \cap \widehat{W}$ for some $x \in \widehat{W}$. The set of tiles will be denoted by $\mathcal{P}_{\widehat{W}}$ and we will call it a tiling of $\widehat{W}$.

Remark. Since $f_{\lambda}$ exchanges the two sets $W_{+}$and $W_{-}$, the definition of the puzzle pieces $P_{x}$ does not depend on the choice of the integer $n$ such that $f_{\lambda}^{\circ n}(x) \in W_{ \pm}$. In particular, if $p_{1} \in \mathcal{P}_{\widehat{W}}$ and $p_{2} \in \mathcal{P}_{\widehat{W}}$ are two tiles, then

$$
\stackrel{\circ}{p_{1}} \cap \stackrel{\circ}{p_{2}}=\emptyset \text {. }
$$


Figure 9 shows the tiling of $\widehat{W}$. The purpose of this section is to give a combinatorial description of this tiling. Notice that since the critical values of $f$ - hence of $f_{\lambda}$ - are contained in $\mathbb{R}$, if $P_{x}$ is a puzzle piece around $x \in \widehat{W}$, then, for $n$ large enough, $f_{\lambda}^{\circ n}: P_{x} \rightarrow W_{ \pm}$is a non-ramified covering, and since $W_{ \pm}$is simply connected, it is an isomorphism. Observe also that $\bar{W}_{+} \cup \bar{W}$ - is a neighborhood of the fixed point $x_{0}$ of $f_{\lambda}$. Hence, every point in the basin $\widehat{W}$ of $f_{\lambda}$ is contained in a tile. In other words,

$$
\widehat{W}=\bigcup_{p \in \mathcal{P}_{\widehat{W}}} p
$$

Besides, the tiling is defined by pulling by the mapping $f_{\lambda}$, but since $f$ is its linearizer, it will give some information about $f$.

Lemma 5. Let $P_{x}$ be a puzzle piece around $x \in \widehat{W}$. Then $f\left(P_{x}\right)=\mathbb{H}_{ \pm}$and $f: P_{x} \rightarrow \mathbb{H}_{ \pm}$is an isomorphism.

Proof. Since the linearizer of $f_{\lambda}$ is the mapping $f$ itself, we can write:

$$
f(z)=\left(-\frac{1}{\lambda}\right)^{n} f \circ f_{\lambda}^{\circ n}(z)
$$

But, if $P_{x}$ is a puzzle piece, there exists an integer $n$ such that $f_{\lambda}^{\circ n}: P_{x} \rightarrow W_{ \pm}$ is an isomorphism (observe also that if $f_{\lambda}^{\circ n}$ is an isomorphism from $P_{x}$ onto $W_{+}$, then $f_{\lambda}^{\circ(n+1)}$ is an isomorphism from $P_{x}$ onto $\left.W_{-}\right)$. Hence,

$$
f\left(P_{x}\right)=\left(-\frac{1}{\lambda}\right)^{n} f\left(W_{ \pm}\right)=\mathbb{H}_{\mp} .
$$

The result follows, since $f: W_{ \pm} \rightarrow \mathbb{H}_{\mp}$ is an isomorphism.

This shows that we could have defined the puzzle pieces as the connected components of $\widehat{W} \backslash f^{-1}(\mathbb{R})$. Hence, if we give a combinatorial description of the tiling, we will at the same time give a combinatorial information on how the critical points of $f: \widehat{W} \rightarrow \mathbb{C}$ are related.

Remark. This won't give any information about the relative position of the critical values.

Observe also that the points that are in several tiles are mapped by $f$ to $\mathbb{R}$. Since $f$ has only simple critical points (Theorem B), any point $z \in \widehat{W}$ belongs to exactly 1,2 or 4 tiles. The points belonging to four tiles are the critical points of $f$.

Lemma 6. Let $p$ be any tile. Then there exists a homeomorphism $\phi_{p}: \mathbb{H}_{+} \cup \mathbb{R} \rightarrow p$, such that $\phi(\mathbb{Z})$ is the set of critical points of $f$ contained in $p$.

Proof. We first claim that for any tile $p$,

- $f: p \rightarrow H_{ \pm} \cup \mathbb{R}$ is a homeomorphism,

- the set $Z$ of critical values of $f \mid p$ is discrete in $\mathbb{R}$, and

- $Z$ accumulates on both $-\infty$ and $+\infty$.

Indeed, this has already been proved in Theorem 4 for the tiles $\bar{W}_{ \pm} \cap \widehat{W}$ : the set of critical values of $f \mid \partial W_{ \pm}$is $\left\{(-1 / \lambda)^{k} \mid k \in \mathbb{N}\right\}$. Now, if $p$ is any tile, we know that $f: \stackrel{\circ}{p} \rightarrow \mathbb{H}_{ \pm}$is an isomorphism (Lemma 5 ), and that there exists an $n$ such that 
$f_{\lambda}^{\circ n}: \stackrel{\circ}{p} \rightarrow W_{ \pm}$is an isomorphism that extends analytically in a neighborhood of $p$. Since

$$
f=\left(-\frac{1}{\lambda}\right)^{n} f \circ f_{\lambda}^{\circ n}
$$

we see that the set $Z$ of critical values of $f \mid p$ is discrete and contains the set $\left\{(-1 / \lambda)^{k} \mid k \geq n\right\}$. Hence it accumulates on both $-\infty$ and $+\infty$.

Next, observe that for any discrete set $Z$ in $\mathbb{R}$ that accumulates both on $-\infty$ and $+\infty$, there exists a homeomorphism $h: \mathbb{H}_{+} \cup \mathbb{R} \rightarrow \mathbb{H}_{+} \cup \mathbb{R}$ mapping $\mathbb{Z}$ to $Z$. Then, the homeomorphism $\phi_{p}=f^{-1} \circ h: \mathbb{H}_{+} \cup \mathbb{R} \rightarrow p$ provides the required homeomorphism.

5.3. Quadratic tilings. We will first define a notion of a quadratic tiling of a simply connected open domain $U \subset \mathbb{C}$. We will then prove that given two quadratic tilings of simply connected domains $U$ and $U^{\prime}$, there always exist a homeomorphism between $U$ and $U^{\prime}$ mapping tiles to tiles.

Definition 11. A quadratic tiling of a simply connected open domain $U \subset \mathbb{C}$ is a set $\mathcal{P}$ of subsets of $U$ such that any point $z \in U$ belongs to exactly 1, 2 or 4 tiles (in particular $U=\bigcup_{p \in \mathcal{P}} p$ ), and for each tile $p \in \mathcal{P}$ there exist a homeomorphism $\phi_{p}: \mathbb{H}_{+} \cup \mathbb{R} \rightarrow p$ such that

- if $z \in \mathbb{H}_{+}$, then $\phi_{p}(z)$ belongs to exactly 1 tile,

- if $z \in \mathbb{Z}$, then $\phi_{p}(z)$ belongs to exactly 4 tiles,

- if $z \in \mathbb{R} \backslash \mathbb{Z}$, then $\phi_{p}(z)$ belongs to exactly 2 tiles, and

- when $z \in \mathbb{H}_{+}$satisfies $|z| \rightarrow+\infty$, then $\phi_{p}(z)$ tends to a point $\phi_{p}(\infty)$ in the boundary of $U$.

We will say that $(U, \mathcal{P})$ is a tiled domain. The points belonging to four tiles will be called "corners", the closure of the connected components of the set of points belonging to exactly two tiles will be called "edges", and the point $\phi_{p}(\infty)$ will be called "the point at infinity in $p$ ".

Remark. This definition has been given so that the tiling $\mathcal{P}_{\widehat{W}}$ is a quadratic tiling of $\widehat{W}$.

Now, given a tiled domain $(U, \mathcal{P})$, a sub-tiling is a subset $P$ of $\mathcal{P}$ such that the union of tiles $p \in P$ is connected and remains connected when the corners are removed. Let us say that $h: p \rightarrow p^{\prime}$ is a tile homeomorphism if $h$ is a homeomorphism between two tiles, respecting the boundary structure, i.e., sending corners and edges of $p$ to corners and edges of $p^{\prime}$. A sub-tiling homeomorphism between two sub-tilings $P$ and $P^{\prime}$ is a homeomorphism

$$
h: \bigcup_{p \in P} p \rightarrow \bigcup_{p^{\prime} \in P^{\prime}} p^{\prime}
$$

such that the restriction to any tile $p \in P$ is a tile homeomorphism. Finally, a tiling homeomorphism between two tiled domains $(U, \mathcal{P})$ and $\left(U^{\prime}, \mathcal{P}^{\prime}\right)$ is a sub-tiling homeomorphism between $\mathcal{P}$ and $\mathcal{P}^{\prime}$.

Proposition 4. Given any two tiled domains $(U, \mathcal{P})$ and $\left(U^{\prime}, \mathcal{P}^{\prime}\right)$ there exists a tiling homeomorphism between them.

Proof. Let $\mathcal{S}$ be the set of sub-tiling homeomorphisms between sub-tilings $P \subset \mathcal{P}$ and sub-tilings $P^{\prime} \subset \mathcal{P}^{\prime}$. This set $\mathcal{S}$ is clearly non-empty, since for any tiles $p \in \mathcal{P}$ 
and $p^{\prime} \in \mathcal{P}^{\prime}$, there is always a tile homeomorphism between $p$ and $p^{\prime}$. Besides, $\mathcal{S}$ can be partially ordered as follows: a sub-tiling homeomorphism $h_{1}$ between $P_{1}$ and $P_{1}^{\prime}$ is larger than a sub-tiling homeomorphism $h_{2}$ between $P_{2}$ and $P_{2}^{\prime}$ if and only if

$$
P_{2} \subset P_{1} \quad \text { and } \quad h_{1} \mid \bigcup_{p \in P_{2}} p=h_{2}
$$

(note that the restriction of $h_{1}$ to any sub-tiling $P \subset P_{1}$ is always a sub-tiling homeomorphism between $P$ and its image). Observe that every totally ordered chain of sub-tiling homeomorphisms $h_{i}, i \in \mathcal{J}$, between sub-tilings $P_{i}$ and $P_{i}^{\prime}$, has an upper bound which is the sub-tiling homeomorphism $h$ between $\bigcup_{i \in \mathcal{J}} P_{i}$ and $\bigcup_{i \in \mathcal{J}} P_{i}^{\prime}$, such that the restriction of $h$ to $P_{i}$ is equal to $h_{i}$. Hence, we can apply Zorn's lemma which shows that there exists a maximal sub-tiling homeomorphism $h$ between two sub-tilings $P \subset \mathcal{P}$ and $P^{\prime} \subset \mathcal{P}^{\prime}$.

If $P=\mathcal{P}$, then the set $\bigcup_{p \in P} p$ contains no edge in its boundary. Since $h$ is a homeomorphism, the same property holds for its image and $P^{\prime}=\mathcal{P}^{\prime}$. Hence, in this case the proof is completed. On the other hand, if $P \neq \mathcal{P}$, then the set $\bigcup_{p \in P} p$ contains an edge $e$ in its boundary. We call $p_{0} \notin P$ the tile that contains $e$ in its boundary and $e^{\prime}$ the image $h(e)$. Since $h$ is a homeomorphism, $e^{\prime}$ is in the boundary of $\bigcup_{p^{\prime} \in P^{\prime}} p^{\prime}$. We call $p_{0}^{\prime} \notin P^{\prime}$ the tile that contains $e^{\prime}$ in its boundary. We will show that we can extend $h$ to a sub-tiling homeomorphism between $P \cup\left\{p_{0}\right\}$ and $P^{\prime} \cup\left\{p_{0}^{\prime}\right\}$.

Let us first show that $\gamma=p_{0} \cap \bigcup_{p \in P} p$ is a connected union of edges, and that $h(\gamma)$ is contained in $p_{0}^{\prime}$. It is obviously a union of edges of $p_{0}$. Assume it is not connected. Then, there exists an edge $e_{1}$ such that the two connected components of $\left(\partial p_{0} \backslash e_{1}\right) \cap U$ both intersect $P$. Moreover, let $p_{1} \notin P$ be the tile which contains $e_{1}$ in its boundary. Then, $p_{0} \cup p_{1}$ separates $U$ in two connected components (because both tiles contain a point at infinity in their boundary), both intersecting $P$. This contradicts the fact that the sub-tiling of $P$ is connected. To see that $h(\gamma)$ is contained in $p_{0}^{\prime}$, observe that for any two edges $e_{1}$ and $e_{2}$ in $\gamma$ satisfying $e_{1} \cap e_{2} \neq \emptyset$, we have the following property: $h\left(e_{1}\right) \in p_{0}^{\prime}$ implies that $h\left(e_{2}\right) \in p_{0}^{\prime}$. Indeed, since $e_{2} \in p_{0}$, it is a boundary edge of $P$, hence its image is a boundary edge of $P^{\prime}$. Let $p_{0}^{\prime \prime} \notin P^{\prime}$ be the tile that contains $h\left(e_{2}\right)$. If we set $x=e_{1} \cap e_{2}$, then $h(x) \in p_{0}^{\prime \prime}$. But, the same argument of connectivity as above shows that $x$ is contained in three tiles of $P$, the fourth tile being $p_{0}$. Hence, there is only one tile which contains $h(x)$ and which is not contained in $P^{\prime}$, and $p_{0}^{\prime \prime}=p_{0}^{\prime}$.

Finally, given two tiles $p \in \mathcal{P}$ and $p^{\prime} \in \mathcal{P}^{\prime}$ and a homeomorphism $h$ between a connected union $\gamma$ of edges in the boundary of $p$, mapping each edge to an edge of $p^{\prime}$, there exists an extension of $h$ to a tile homeomorphism $\tilde{h}: p \rightarrow p^{\prime}$. This finishes the proof of the proposition.

5.4. The tiling for a Blaschke product. Let $B(z)$ be a Blaschke product

$$
\left.B(z)=z \frac{z-a}{1-a z}, \quad a \in\right] 0,1[\text {. }
$$

Then $B$ has a fixed point at 0 with multiplier $-a$. The basin of this critical point is $\mathbb{D}$ and contains only one simple critical point $\omega \in \mathbb{D}$. It is a classical result due to Fatou that if $\psi: \mathbb{D} \rightarrow \mathbb{C}$ is a linearizer of the Blaschke product

$$
\left.B(z)=z \frac{z-a}{1-a z}, \quad a \in\right] 0,1[,
$$


then $\psi$ is a ramified covering map, ramified at the inverse images of the critical point $\omega$.

Figure 10 shows a tiling $\mathcal{P}_{B}$ associated to the Blaschke product $B$. The tiles are the closure in $\mathbb{D}$ of the connected components of $\mathbb{D} \backslash \psi^{-1}(\mathbb{R})$.

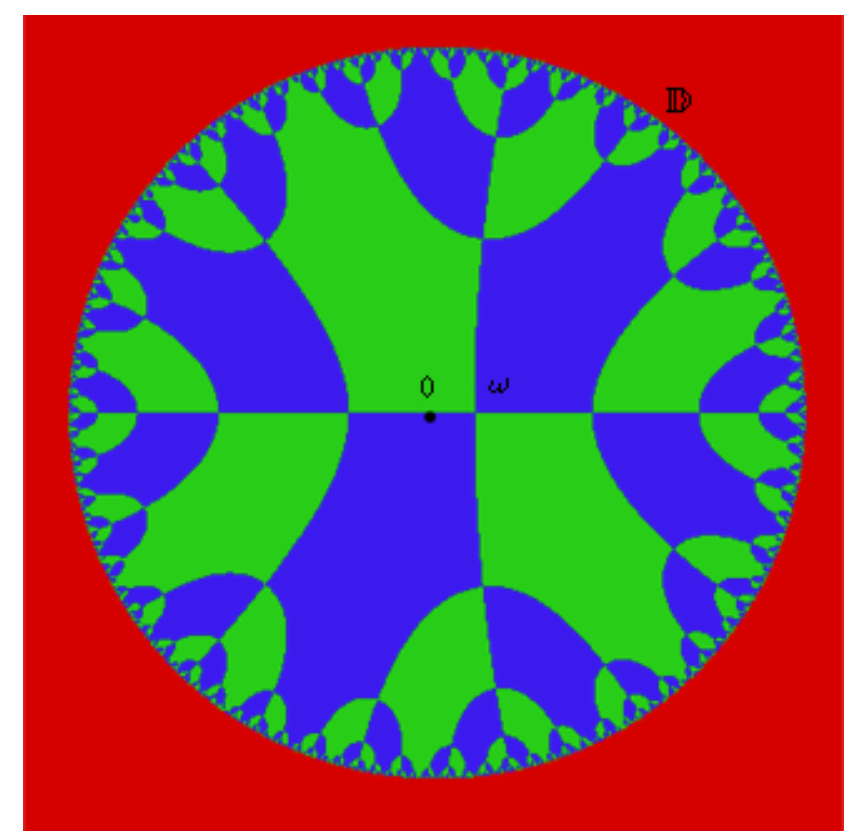

Figure 10. The linearizer $\psi$ which is tangent to the identity at 0 maps blue regions to $\mathbb{H}_{-}$and green regions to $\mathbb{H}_{+}$.

The critical points of $\psi$ are the preimages by $B$ of the critical point $\omega$ of $B$ contained in $\mathbb{D}$. They are simple critical points. Hence, given a point $z \in \mathbb{D}$, we have three possibilities:

- $\psi(z) \in \mathbb{H}_{ \pm}$and $z$ belongs to exactly one tile,

- $z$ is a critical point of $\psi$ and $z$ belongs to exactly four tiles since $\psi$ has only simple critical points,

- $z$ is not a critical point of $\psi$ and $\psi(z) \in \mathbb{R}$, and $z$ belongs to exactly two tiles.

Besides, $\psi$ maps each tile $p \in \mathcal{P}_{B}$ to $\mathbb{H}_{ \pm}$homeomorphically. The set of critical values of $\psi \mid p$ is of the form $\left\{\psi(\omega) / a^{k}\right\}_{k \geq k_{0}}$, for some $k_{0} \geq 0$. Hence the image of the corners form a discrete set in $\mathbb{R}$ accumulating on both $+\infty$ and $-\infty$. We can then show, as in the case of the tiling $\mathcal{P}_{\widehat{W}}$, that the tiling $\mathcal{P}_{B}$ is a quadratic tiling. Hence, by Proposition 4 there exists a homeomorphism between $\mathbb{D}$ to $\widehat{W}$ mapping the tiles of $\mathcal{P}_{B}$ to the tiles of $\mathcal{P}_{\widehat{W}}$.

\section{Topology of the Boundary of $\widehat{W}$}

The domain $\widehat{W}$ is dense in $\mathbb{C}$, hence its boundary has empty interior. However, it seems to have a very complex structure. Studying its topology, its Hausdorff dimension, or its Lebesgue measure could be interesting. But we do not have many 
tools at the moment. However, we will show the surprising result that there are bi-accessible points in the boundary of $\widehat{W}$.

First of all, remember that Theorem 4 tells that the boundary of $\partial W_{+}$can be decomposed as follows.

Definition 12. The boundary $\partial W_{+}$is the union of

- an arc $\gamma_{1}$ which is mapped by $f$ to $\mathbb{R}_{+}$,

- an arc $\gamma_{2}$ which is mapped by $f$ to $\mathbb{R}_{-}$, and

- a point $x_{1}$ such that $\left(x_{1}\right)^{2}=F^{-1}(-i \infty)$.

Theorem C. The point $x_{1} / \lambda$ belongs to the boundary of $\widehat{W}$, and is accessible from $\widehat{W}$ with at least three accesses.

Proof. We claim that $\left(x_{1}, \bar{x}_{1}\right)$ is a repelling cycle of period 2 for the map $f_{\lambda}$. Indeed, $f_{\lambda}^{\circ 2}$ is an automorphism of $W_{+}$(see Lemma 1 ). Since there is an attracting fixed point in the boundary (the point $x_{0}$ ), there must also be a repelling fixed point on the boundary. But the only point in $\partial W_{+}$which is not in the basin $\widehat{W}$ is the point $x_{1}$. Hence, $x_{1}$ is a repelling fixed point for $f_{\lambda}^{\circ 2}$. Since $f_{\lambda}$ maps $W_{+}$to $W_{-}$, $x_{1}$ cannot be a fixed point of $f_{\lambda}$. This shows that $x_{1}$ is a repelling periodic point of period 2 for $f_{\lambda}$. Since the map $f_{\lambda}$ is a real map, the other point of the cycle is $\bar{x}_{1}$.

In particular, $x_{1}$ does not belong to the basin $\widehat{W}$, and it is in its boundary. Moreover, according to Lemma $2, \lambda \widehat{W} \subset \widehat{W}$. Hence $\lambda^{-1} \partial \widehat{W} \subset \partial \widehat{W}$, so that $x_{1} / \lambda \in$ $\partial \widehat{W}$.

The Feigenbaum map $f$ maps $\gamma_{1}$ and $\gamma_{2}$ to $\mathbb{R}_{+}$and $\mathbb{R}_{-}$, which are contained in $\widehat{W}$. Hence, $f_{\lambda}$ maps $\gamma_{1}^{\prime}=\gamma_{1} / \lambda$ and $\gamma_{2}^{\prime}=\gamma_{2} / \lambda$ inside its basin of attraction $\widehat{W}$. Moreover, the imaginary axis is also contained in $\widehat{W}$. Let us call $\gamma_{3}^{\prime}$ its inverse image by $f_{\lambda} \mid W_{+}$. The $\operatorname{arcs} \gamma_{1}^{\prime}, \gamma_{2}^{\prime}$ and $\gamma_{3}^{\prime}$ are in the basin of attraction of $f_{\lambda}$, i.e., in $\widehat{W}$ (see Figure 9 ), and they land at the same point $x_{1} / \lambda$ which is "mapped" by $f$ to $-i \infty$.

We must show that those arcs do not define the same access to $x_{1} / \lambda$. This condition is not difficult to check (see Figure 11). Those arcs cut $W_{+} / \lambda$ in two pieces which are mapped by $f_{\lambda}$ to the two quarter-planes contained in $\mathbb{H}_{-}$. Hence, each one of those pieces contains a point which is not contained $\widehat{W}$ (either $x_{1}=f_{\lambda}^{-1}\left(\bar{x}_{1}\right)$ or $\left.f_{\lambda}^{-1}\left(-x_{1}\right)\right)$.

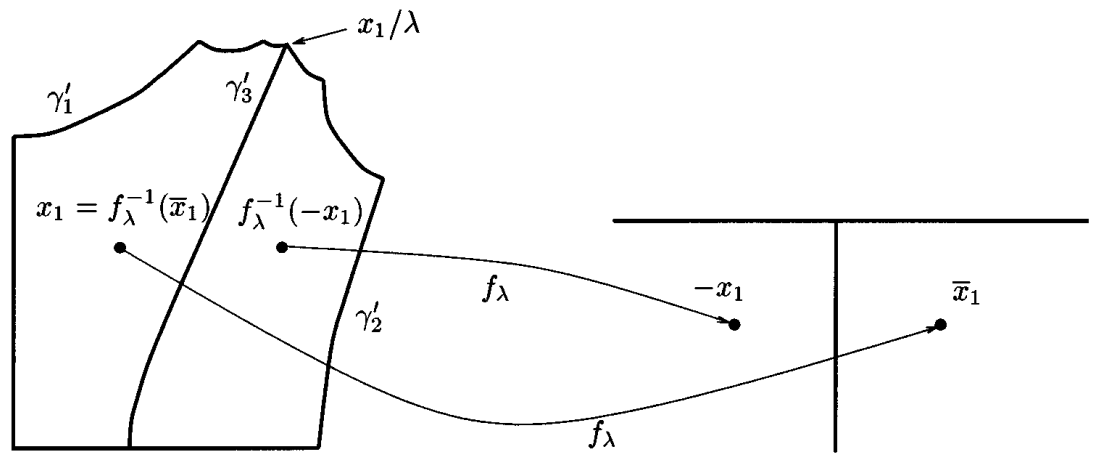

Figure 11. The $\operatorname{arcs} \gamma_{1}^{\prime}, \gamma_{2}^{\prime}$ and $\gamma_{3}^{\prime}$. 


\section{Local CONNECTIVITY OF $K(f)$}

Definition 13. We define puzzles $\mathcal{P}_{n}, n \in \mathbb{N}$ in the following way:

- the puzzle pieces of the puzzle $\mathcal{P}_{0}$ of depth 0 are the puzzle pieces $P_{x}$ defined in Definition 10, and

- the puzzle pieces of the puzzle $\mathcal{P}_{n}$ of depth $n$ are the pieces $\lambda^{n} P, P \in \mathcal{P}_{0}$.

Figure 12 shows some pieces of the puzzles of depth 1,2, and 3 . We have colored the puzzle pieces of depth 2 in blue and green and the puzzle pieces of depth 3 in brown and yellow. A lot of pieces are missing, but drawing them takes a bit of time. That is why the region covered by the puzzle pieces seem to be disconnected, which is not the case: remember that the tiles cover a dense open subset $\widehat{W} \subset \mathbb{C}$ which is connected and simply connected (see Figure 9).

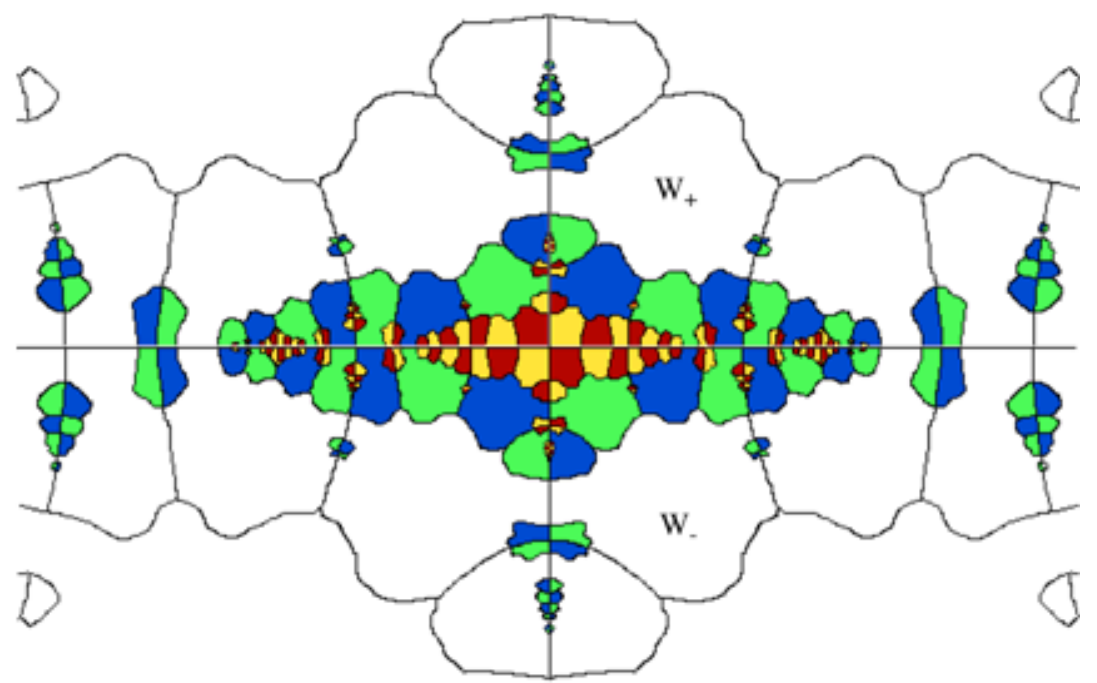

Figure 12. Some pieces of the puzzles $\mathcal{P}_{i}, i=1,2,3$.

Since the closure of the puzzle pieces of depth $n$ cover the set $\lambda^{n} \widehat{W}$, and since $K(f) \subset \lambda^{n} \widehat{W}$ for every $n \geq 0$ (see Lemma 3 ), we see that the closure of the puzzle pieces of depth $n$ cover the Julia set $K(f)$. We will first show that the intersection of the closure of a puzzle piece with $K(f)$ is always connected (Lemma 7), and then we will prove that the puzzle of depth $n$ subdivides the puzzle of depth $n-1$ (Lemma 8).

Lemma 7. If $P$ is a puzzle piece, then for all $n \geq 0$, the intersection $\lambda^{n} \bar{P} \cap K(f)$ is connected.

Proof. Given a puzzle piece $P$, there exists an integer $k \geq 0$ such that $f_{\lambda}^{k}(P)=W_{ \pm}$. We will call this $k$ the age of the puzzle piece, and we will prove this lemma by induction on the age of the puzzle piece $P$.

For $k=0, \lambda^{n} \bar{W}_{ \pm} \cap K(f)$ is connected, because $f^{\circ 2^{n}}\left(\lambda^{n} \bar{W}_{ \pm}\right)$is a half-plane, which cuts $K(f)$ in two parts along the real axis, and because $K(f)$ is totally invariant by the polynomial-like map $f: W \rightarrow \mathbb{C}_{\lambda}$. 
If $P$ is a puzzle piece of age $k+1 \geq 1$, we first notice that $K(f) \subset W$. Hence,

- either $\lambda^{n} P \cap W=\emptyset$, and $\lambda^{n} P \cap K(f)=\emptyset$ is connected,

- or $\lambda^{n} P \subset W$.

On the other hand, $f_{\lambda}(P)=Q$ is a puzzle piece of age $k$, and by inductive hypothesis, every $\lambda^{n} \bar{Q} \cap K(f), n \in \mathbb{N}$, is connected. Besides,

$$
f(\lambda P)=\left(-\frac{1}{\lambda}\right)^{n-1} f^{\circ 2^{n-1}}\left(\lambda^{n-1} \lambda P\right) .
$$

Hence,

$$
f^{\circ 2^{n-1}}\left(\lambda^{n} P\right)=(-\lambda)^{n-1} f_{\lambda}(P)=(-\lambda)^{n-1} Q .
$$

Since $K(f)$ is totally invariant by $f \mid W$, we can conclude that $\lambda^{n} \bar{P} \cap K(f)$ is also connected when $\lambda^{n} P \subset W$.

We can now show that the puzzle $\mathcal{P}_{n+1}$ subdivides the puzzle $\mathcal{P}_{n}$. In other words, we will show the following lemma.

Lemma 8 (see Figure 12). Every puzzle piece $\lambda P \in \mathcal{P}_{1}$ is contained in a puzzle piece $Q \in \mathcal{P}_{0}$.

Proof. Let $z$ be a point in $\lambda P$ and let $Q$ be the puzzle piece which contains $z$. Using Lemma 5 , we see that $f(Q)=\mathbb{H}_{ \pm}$. Since $f(\lambda P)=f_{\lambda}(P)$ is a puzzle piece (by definition), and since puzzle pieces do not intersect the real line (they are the connected components of $\widehat{W} \backslash f^{-1}(\mathbb{R})$, and $\mathbb{R} \subset f^{-1}(\mathbb{R})$ ), we know that

$$
f(\lambda P) \subset \mathbb{H}_{ \pm}=f(Q),
$$

and as $f: Q \rightarrow \mathbb{H}_{ \pm}$is an isomorphism, we can deduce that

$$
\lambda P \subset Q .
$$

This construction makes it possible to recover the following result due to $\mathrm{Hu}$ and Jiang $[\mathrm{HJ}]$.

Theorem 5 (see Figure 13). The Julia set $K(f)$ is locally connected at the critical point.

Up to now, we can only prove local connectivity at the critical point 0 :

- $0 \in \lambda^{n} W \subset \lambda^{n-1} W$, and

- $\lambda^{n} \bar{W} \cap K(f)$ is connected.

One can use classical arguments of recurrence to spread around this local connectivity. However, those arguments do not exhibit a nice basis of connected neighborhoods. We would like to prove that if we consider the nested sequence of puzzle pieces $P_{n}(z) \in \mathcal{P}_{n}$ which contain a point $z \in K(f)$, then the diameter of the piece $P_{n}(z)$ decreases geometrically with $n$. We just need to show the following conjecture.

Conjecture 1. There exists a constant $\varepsilon>0$ such that if $P \in \mathcal{P}_{0}$ and $Q \in \mathcal{P}_{0}$ are puzzle pieces and $\lambda P \subset Q$, then

$$
\frac{\operatorname{diam}(Q)}{\operatorname{diam}(\lambda P)} \geq 1+\varepsilon
$$

We think it is possible to show this conjecture using some classical arguments of moduli of annuli. Moreover, we think that there is a stronger result. 


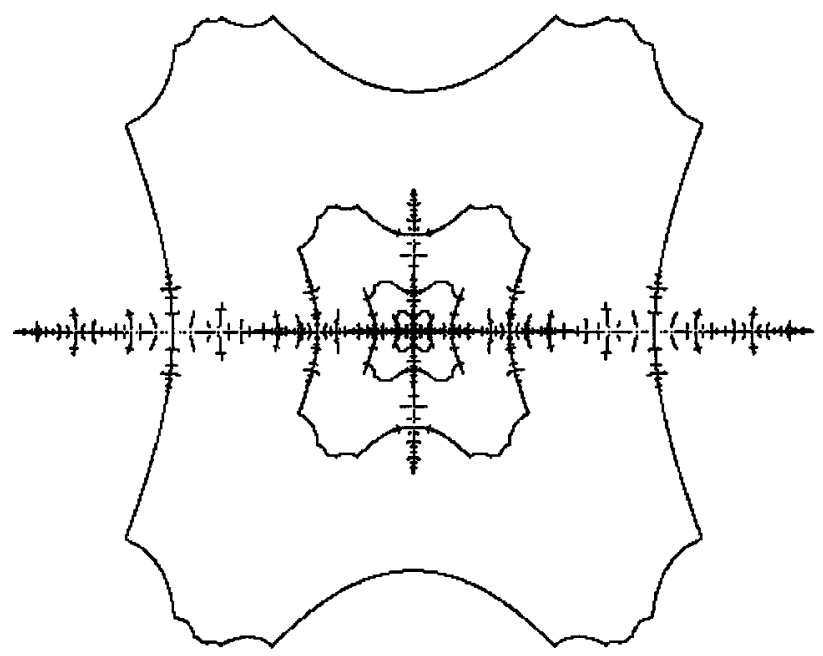

Figure 13. Local connectivity of the Feigenbaum Julia set at the critical point.

Conjecture 2. There exists a constant $K \in \mathbb{R}$ such that the diameter of every puzzle piece $P \in \mathcal{P}_{0}$ is bounded by $K$ :

$$
\operatorname{diam}(P) \leq K
$$

\section{REFERENCES}

[CT] P. Coullet and C. Tresser, Itération d'endomorphismes et groupe de renormalisation, J. Phys. Colloque C 539, C5-25 (1978).

[DH] A. Doundy and J.H. Hubbard, On the Dynamics of Polynomial-like Mappings, Ann. Scient., Ec. Norm. Sup. $4^{e}$ series, vol. 18, (1985), 287-343. MR 87f:58083

[EW] J.P. Eckmann and P. Wittwer, A complete proof of the Feigenbaum conjectures, J. Stat. Phys., vol 46 (1987), 455-477. MR 89b:58131

[E1] H. EpSTEIn, Fixed points of composition operators II. Nonlinearity, vol. 2, (1989), 305310. MR 90j:58086

[E2] H. EPSTEIN, Fixed points of the period-doubling operator, Lecture notes, Lausanne.

[F1] M.J. FeIgenbaum, Quantitative universality for a class of non-linear transformations, J. Stat. Phys., vol. 19, (1978), 25-52. MR 58:18601

[F2] M.J. Feigenbaum, The universal metric properties of non-linear transformations, J. Stat. Phys., vol. 21, (1979), 669-706. MR 82e:58072

[HJ] J. Hu and Y. JIANG, The Julia set of the Feigenbaum quadratic polynomial is locally connected, Preprint, (1993).

[L] O.E. LANFORD A computer assisted proof of the Feigenbaum conjectures, Bull. Amer. Math.Soc., vol. 6, (1982), 427-434. MR 83g:58051

[McM] C.T. McMullen, Renormalization and 3-manifolds which fiber over the circle, Annals of Math Studies, Princeton University Press, vol. 142, (1996). MR 97f:57022

[dMvS] W. De Melo and S. van Strien, One dimensional dynamics, Springer-Verlag, (1993). MR 95a:58035

[S] D. Sullivan, Bounds, quadratic differentials and renormalization conjectures, In F. Browder, editor, Mathematics into Twenty-first Century: 1988 Centennial Symposium, August 8-12, Amer. Math. Soc., (1992), 417-466. MR 93k:58194

Université Paul Sabatier, Laboratoire Emile Picard, 31062 Toulouse cedex, France 